\title{
CMF-DFE Based Adaptive Blind Equalization Using Particle Swarm Optimization
}

\author{
Emin TUGCU, Ismail KAYA, Ayhan YAZGAN \\ Dept. of Electrical-Electronics Engineering, Karadeniz Technical University, Trabzon, Turkey \\ emintugcu@ktu.edu.tr, ikaya@ktu.edu.tr, ayhanyazgan@ktu.edu.tr \\ Manuscript received September 2, 2015
}

\begin{abstract}
The channel matched filter $(C M F)$ is the optimum receiver providing the maximum signal to noise ratio (SNR) for the frequency selective channels. The output intersymbol interference (ISI) profile of the CMF convolved by the channel can be blindly obtained by using the autocorrelation of the received signal. Therefore, the inverse of the autocorrelation function can be used to equalize the channel passed through its own CMF. The only missing part to complete the proposed blind operation is the CMF coefficients. Therefore, in this work, the best training algorithm investigation is subjected for blind estimation of the CMF coefficients. The proposed method allows using more effective training algorithms for blind equalizations. However, the expected high performance training is obtained when the swarm intelligence is used. Unlike the stochastic gradient algorithms, the particle swarm optimization (PSO) is known to have fast convergence because its performance is independent of the characteristics of the systems used. The obtained mean square error (MSE) and bit error rate (BER) performances are promising for high performance real-time systems as an alternative to non-blind equalization techniques.
\end{abstract}

\section{Keywords}

Blind channel estimation, blind channel equalization, particle swarm optimization, channel matched filter

\section{Introduction}

Mitigating the delay spread of the multipath channel is a challenging equalization task that requires significant processing power and effective training algorithms. Unless the communication frequency band is divided in small parts as it is the case in the orthogonal frequency division modulation (OFDM), the ISI nature of a channel has to be dealt as a finite impulse response (FIR) [1] and resolved either by one of complex Markov chain analysis methods as in Viterbi Algorithm (VA) or filtered by a channel inverting filter which is called equalizer. In order to implement any of these mentioned methods, either channel coefficients or some part of the transmitted data called training sequence, should be known by the receiver. In practice, a training sequence is placed at the beginning of the transmitted data packet. The training sequence guides to obtain the channel coefficients or is used to train the channel equalizer. On the other hand, the training sequence is not desirable for two main reasons. First, it degrades the throughput level and secondly it increases the implementation complexity because of its pre-requisites. Therefore, as it was a call by Labat saying that "Can you skip the training period?" an algorithm is required implementing the ISI cancellation without using any known symbols, such as training sequence [2].

As it is mentioned in [3], [4], there are some applications where training sequence cannot be used. Therefore, blind training techniques are emerged to train the equalizer without any cooperation between the transmitter and the receiver. In blind trainings there is no knowledge about the communication medium; only available information at the receiver is the statistical data obtained from the received signal.

One of the early contributions to blind equalizations was made by Sato [5] and it was followed by several studies in order to improve training performance of blind algorithms. In the literature a linear transversal equalizer (LTE) is used commonly for blind training studies [5]-[7]. Due to error propagation of the feedback filter, the decision feedback equalizer (DFE) has been mostly avoided in studies [8]. Nevertheless, there has been some research on blind trainings of DFE as its performance is better than LTE [9]-[11]. The most inspiring work on the area of blind training was published by Godard, by his proposition called Constant Modulus Algorithm (CMA) [6]. The CMA offers low complexity and reasonable stable convergence. However, most of the time it is found slow and its error level is high, considering the high performance required applications. Therefore several studies have been concentrated on improving the performance of CMA [4], [12]-[15].

There are other types of blind training algorithms, such as using second order statistic or Fourier analysis of incoming data. The blind equalization techniques which uses second or higher degrees moments of the received signals are presented in [16], [17]. The method which estimates data and channel by using the maximum likelihood approach is studied in [18]. For example, in studies [19], [20], Baykal implemented an effective blind LTE and DFE trainings using Fourier analysis to obtain the matched filter 
of the channel. The reference [19] is the first study using CMA algorithm in order to directly obtain the channel impulse response (CIR). In [20] the amplitude response of the channel is obtained blindly by using the autocorrelation of the channel output. In that study, the blind equalizer is used for the estimation of the phase response of the channel. Another work presents a simple recursive algorithm for matrix inversing and matrix multiplication operations for the match filter based blind receiver [21]. A CMF-DFE based blind channel estimation and equalization method is presented in [22].

In this study, instead of implementing blind equalization which is non-convex in essence, we propose a new method that implies the blind training for estimating the CIR coefficients where the operations are convex. The proposed method uses the CMF and DFE features exploited using the second order analysis. For the proposed algorithm the error function has been changed into estimation error form using the channel output. Therefore, the proposed method allows the usage of conventional training algorithms, such as least mean square (LMS) or recursive least squares (RLS) algorithms. Another contribution of this study is to use particle swarm optimization (PSO) [23], which has a superior convergence speed than LMS in trainings and offers much simpler algorithm than RLS. Therefore, the convergence speed of the proposed method is as good as any training sequence based algorithm. In order to demonstrate the performance of the proposed blind algorithm, BER and MSE analysis have been carried out by simulation of frequency selective channels.

The outline of the paper is as follows: In Sec. 2, the proposed CMF based DFE and its blind training equations are introduced. Section 3 explains the PSO algorithm and its applications to blind trainings. Section 4 compares MSE and BER performances of the conventional and proposed algorithms. And, finally we draw the conclusion of the paper in Sec. 5 .

\section{The System Model of Blind CMF- DFE}

The output signal of a typical communication channel can be represented with the convolution $v(t)=$ $\sum_{n=-\infty}^{\infty} x(n) h\left(t-n T_{\mathrm{s}}\right)+\eta(t)$, where $x(n)$ is the transmitted symbol, $h(t)$ is the continuous time channel impulse response (CIR), $\eta(t)$ represents the Additive White Gaussian Noise (AWGN) and $T_{\mathrm{s}}$ is the symbol period. Then, the equivalent discrete time model is $v(k)=$ $\sum_{i=-\infty}^{\infty} h(i) x(k-i)+\eta(k)$. In the paper, it is assumed that the symbols $x(k)$ are independent and identically distributed with a variance of $\sigma_{\mathrm{x}}^{2}$, the noise $\eta(k)$ is uncorrelated with the input symbols having a variance $\sigma_{\eta}{ }^{2}$. It is assumed that the channel is causal with finite memory of length $L$ and transmit-receive filters do not create ISI. Finally, the output of the channel is given as follows

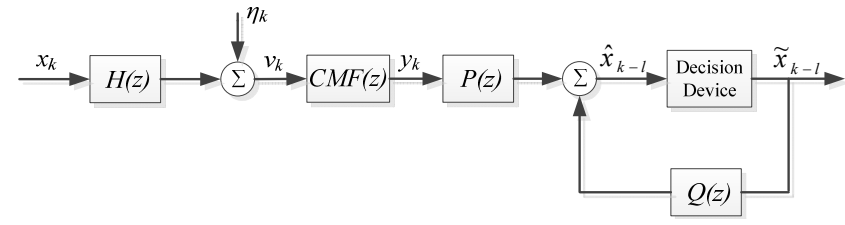

Fig. 1. The block diagram of the CMF-DFE.

$$
v_{k}=\sum_{i=0}^{L} h_{i} x_{k-i}+\eta_{k}
$$

Figure 1 shows the block diagram of the CMF-DFE receiver where $C M F(z), P(z)$ and $Q(z)$ represent the impulse responses of $\mathrm{CMF}$, feed-forward and feedback filters of the DFE, respectively. The $C M F(z)$ is the matched filter of channel $H(z)$, having its mirror image as $m_{-i}=h_{i}{ }^{*}$, $i=0, \ldots, L$ when $h_{i}$ 's are the channel coefficients.

The CMF-DFE provides several advantages over the conventional DFE structure [24], [25]. In [24], static calculation of CMF-DFE which provides quite stable and high performance equalizer implementation is studied. They are calculated blindly by using the autocorrelation function of incoming sequence at the receiver, and run the blind trainings for the CMF which is a linear filter. The impulse response of CMF is given by (2) and (3)

$$
\begin{gathered}
C M F(z)=H^{*}\left(1 / z^{*}\right), \\
m_{-i}=h_{i}^{*}, i=0, \ldots, L .
\end{gathered}
$$

In order to obtain maximum energy at the input of equalizer filter, the size of CMF has to be equal to the size of channel, as it is the case in (3). The ISI components $d_{k}$, $k=-L, \ldots, L$, at the output of the CMF can be calculated as shown in (4).

$$
d_{k}=\sum_{i=0}^{L-k} h_{i}^{*} h_{i+k}, k=0, \cdots, L
$$

where the center ISI component of (4) is given in (5).

$$
d_{0}=h_{L}^{*} h_{L}+h_{L-1}^{*} h_{L-1}+\cdots+h_{1}^{*} h_{1}+h_{0}^{*} h_{0} .
$$

In (5) $d_{0}$ is a real largest component. This center tap combines all of the multipath energy of the channel. In (4) the ISI components are symmetrical and complex conjugates on both sides of the center tap, $d_{0}$ as shown in (6)

$$
d_{-k}=d_{k}^{*}, k=1, \cdots, L .
$$

Therefore, using (1) the output of CMF is given as

$$
y_{k}=\sum_{i=0}^{L} h_{L-i}^{*} v_{k-i} .
$$

Using the final ISI components $d_{i}$ 's, (7) becomes as given in $(8)$

$$
y_{k}=\sum_{i=-L}^{L} d_{i} x_{k-i}+\eta_{k}^{\prime} .
$$

If the channel is known by the receiver, the ISI components of CMF are calculated by (4) as in [25]. However, if the channel coefficients are unknown for the receiver and 
there is no cooperation between the transmitter and the receiver, the ISI component can be calculated by (9).

$$
d_{j}=E\left\{v_{k} v_{k-j}^{*}\right\}
$$

where $E\{\cdot\}$ shows the expectation operator. Additionally, (9) also represents the calculation of the autocorrelation coefficients of channel transfer function, blindly. The output of DFE is calculated by

$$
\hat{x}_{k}=\sum_{n=-L_{\text {ff }}}^{0} c_{n} y_{k-n}+\sum_{m=1}^{L_{\mathrm{fb}}} c_{m} \tilde{x}_{k-m}
$$

where $c_{i}$ 's are equalizer coefficients, $\hat{x}_{k}$ and $\tilde{x}_{k}$ are the estimated and detected data at the output of equalizer, respectively. The constants $L_{\mathrm{ff}}$ and $L_{\mathrm{fb}}$ are the number of feedforward and feedback tap numbers of DFE filter. When a CMF filter is assumed available before equalizer, the DFE coefficients can be directly calculated by the zeroforcing method [24], [26] as

$$
\begin{aligned}
& \mathbf{D} \times \mathbf{c}=\mathbf{b} \\
& \text { when } \\
& \mathbf{c}=\left[\begin{array}{lllll}
c_{-L_{\mathrm{ff}}} & \ldots & c_{0} & \ldots & c_{L_{\mathrm{fb}}}
\end{array}\right]^{T}, \mathbf{b}=\left[\begin{array}{lllll}
0 & \cdots & 1 & \cdots & 0
\end{array}\right]^{T}
\end{aligned}
$$
and

$$
\mathbf{D}=\left[\begin{array}{cccccccc}
d_{0} & d_{1} & \cdots & d_{L} & 0 & 0 & \cdots & 0 \\
d_{1}^{*} & d_{0} & \cdots & d_{L-1} & d_{L} & 0 & \cdots & 0 \\
\vdots & \vdots & \cdots & \vdots & \vdots & \vdots & \cdots & \vdots \\
d_{L}^{*} & d_{L-1}^{*} & \cdots & d_{0} & d_{1} & 0 & \cdots & 0 \\
0 & d_{L}^{*} & \cdots & d_{1}^{*} & d_{0} & 0 & \cdots & 0 \\
0 & 0 & \cdots & d_{2}^{*} & d_{1}^{*} & 1 & \cdots & 0 \\
\vdots & \vdots & \cdots & \vdots & \vdots & \vdots & \cdots & \vdots \\
0 & 0 & \cdots & 0 & d_{L}^{*} & 0 & \cdots & 1
\end{array}\right] .
$$

The Toeplitz matrix equation is given in (11), and can be easily solved by numerical techniques, such as Levinson-Durbin algorithm [25], [26]. The components of D matrix are calculated by (9) using only the received data. Equation (11) also corresponds to the Minimum Mean Square Error (MMSE) equalizer as in [27], providing that the CMF presents before DFE [24], [28]. In Fig. 1, the channel coefficients are unknown for the receiver. However, the ISI components at the output of the CMF (which is not the transfer function of the CMF) and DFE coefficients are calculated by (9) and (11). Therefore, a blind training is only required to estimate the CMF coefficients as shown in Fig. 1.

\section{Particle Swarm Optimization Algorithm}

PSO algorithm is one of the popular population based evaluation type optimization algorithm which has been developed by James Kennedy and Russell Eberhart [23]. The algorithm has been found useful for stochastic based optimizations and takes place in many industrial applica- tions [29], [30]. PSO algorithm has high convergence rate, low complexity profile and is successful in avoiding local minimums. PSO uses swarm intelligence to provide different solutions. The solution space is the constrained with the size of $M$. For the $i$ th particle, the solution set is given by $\mathbf{p}_{i}=\left[p_{i 1} p_{i 2} \ldots p_{i M}\right]^{T}$. The initial values of every particle are randomly selected in order to start searching the optimum point. Every particle has two components which are shown by position $(\boldsymbol{p})$ and speed $(\boldsymbol{u})$ vectors. Position vector contains the position state and the speed vector holds the speed and direction of the particle. The speed and position vectors are obtained by the experiences from the previous iterations using both the best performance of the particle (called personal best, $p b e s t$ ) and best performance observed within all particles (called global best, gbest) as in (12) and (13) [23].

$$
\begin{gathered}
u_{i d}=u_{i d}+c_{1} r_{1}\left(\text { pbest }_{i d}-p_{i d}\right)+c_{2} r_{2}\left(\text { gbest }_{d}-p_{i d}\right), \\
p_{i d}=p_{i d}+u_{i d} \quad 1 \leq d \leq M
\end{gathered}
$$

where $c_{1}$ and $c_{2}$ are the acceleration coefficients which drive the particle towards the pbest and gbest values. $r_{1}$ and $r_{2}$ are two random variables uniformly distributed within 0 to 1 . The implementation of PSO is given below;

1. The problem space (allowable maximum and minimum values) is defined.

2. All particles randomly placed into the defined problem space.

3. Positions of particles are controlled whether they are in the problem space or not. If any particle is outside the problem space then a random position is assigned for the particular particle.

4. The fitness function of each particle is calculated.

5. The pbest value of every particle is controlled with its previous value, if the new value is better than previous one, then pbest is updated and algorithm is continued. Otherwise, the previous pbest value is kept.

6. The best pbest value of all particles is attained as the gbest value.

7. If the new gbest is better than the previous gbest, the gbest value is updated by the new one, but if it is not, then the previous gbest value is kept.

8. New speeds and positions of particles are calculated by (12) and (13) respectively.

9. The algorithm is continued from step 3, unless an acceptable error limit has been obtained.

\section{The Proposed MF-DFE Blind Equalizer}

Fast convergence rate of PSO has made PSO algorithm be used in time limited applications and considered as a potential tool for optimization problems for complex engineering applications [31], [32]. Another advantage of 
PSO is that it is not sensitive to parameter selection and does not involve with any differential equation [23]. The non-convex profile of error functions of stochastic based algorithms, like equalizer filter, suffers from slow convergence and low performance. On the other hand, if the stochastic process is required to operate blindly, the convergence problem rises quickly. Multiple solutions space of PSO offers quite high convergence and avoids remaining in a local minimum by its information transfers between particles. Therefore, in this study the PSO is chosen for the required blind training algorithm and found to be good candidate for practical applications thanks to its high performance and fast convergence profiles. Fig. 2 shows the proposed blind equalizer architecture. During the blind training the switch is closed to "1" side. Since the components of $\boldsymbol{D}$ matrix in (11) are autocorrelation products of the channel and they can be calculated blindly by using (9), then the component of $\boldsymbol{b}$ vector corresponding to cursor point is equal to 1 and all other components of $\boldsymbol{b}$ vector are equal to zero in (11). Therefore, the equalizer coefficients $\left(c_{i}\right)$ are directly calculated by solving (11). The only missing part in Fig. 2 is the CMF coefficients which are equal to channel coefficients to be trained blindly. Equation (11) implies the zero-forcing rule by placing unit gain for cursor symbol and zero gain for all other symbols involved in DFE equation in (10), as in [28]. Thus, the non-convex convergence profile of an equalizer is avoided by only remaining channel coefficients to be estimated, which is turned to be linear filter estimation in order to complete the receiver. In addition, CMF plays a crucial role in equalization by providing a uniform ISI profile and accurate synchronization [24]-[28]. However, the equalization problem has been significantly simplified. The required filter coefficients, CMF components, are equal to channel coefficients as in relation stated by

$$
\hat{m}_{-i}=\hat{h}_{i}^{*}, i=0, \ldots, L^{\prime}
$$

where $L^{\prime}$ is the length of the estimated channel filter which is greater than the actual size of the channel, $L$, and $\hat{m}_{i}^{\prime}$ s are the CMF coefficients to be estimated. The output of CMF is given by

$$
\hat{y}_{k}=\sum_{i=0}^{L^{\prime}} \hat{h}_{L^{\prime}-i}^{*} v_{k-i}
$$

At the output of the detector, an estimate of the detected symbol $\tilde{x}_{k}$ would be fed into the feedback section of the
DFE. The detector implementation depends on the modulation type (if it is known at the receiver).

Alternatively, instead of using the detected data in the feedback filter, the estimated soft data can be used instead. In this case, there would be no degradation in ISI cancellation and any unknown modulation data would be equalized. However, in this case, the data would be aligned in an arbitrary direction as mentioned in [33] and slower convergence in parameter estimation would be observed. For faster convergence, a soft limiter, as defined by (16) and (17), can be used. The output of the soft limiter is defined as

$$
\tilde{x}_{k}=\gamma_{k} \angle \hat{\theta}_{k}
$$

where $\hat{\theta}_{k}=\arg \left(\hat{x}_{k}\right)$, which is phase angle of the estimated data $\hat{x}_{k}$, and $\gamma_{k}$ is the amplitude of the soft limiter output.

$$
\gamma_{k}=\left\{\begin{array}{cc}
1 & \text { if }\left|\hat{x}_{k}\right|>1 \\
\left|\hat{x}_{k}\right| & \text { otherwise }
\end{array}\right.
$$

After obtaining the estimates of the incoming signal as given in (18), an error function based on the received data can be set as given in (19).

$$
\begin{aligned}
\hat{v}_{k-l} & =\sum_{i=0}^{L^{\prime}} \hat{h}_{i} \tilde{x}_{k-i-l}, \\
\varepsilon_{k} & =v_{k}-\hat{v}_{k-l}
\end{aligned}
$$

where $v_{k}$ is the output of the channel, $L^{\prime}$ is the expected maximum number of interfering symbols in the channel and $l$ is a fixed delay pointing the cursor symbol. The best choice for $l$ is equal to $L_{\mathrm{ff}}+L^{\prime}-1$ where -1 is for the nondelayed tap at the input of matched filter. It is better to choose $L^{\prime} \geq L$ since the symbol span of the estimated CMF should be equal or greater than the symbol span of the channel. However, the recovery process of the proposed equalizer starts from an arbitrary symbol synchronization point, which initially coincides with any symbol at the any tap of the estimated CMF filter. Therefore, best choice for the length of the estimated CMF is $L^{\prime}=2 L+1$ which is the resulted symbol span of the autocorrelation of channel, calculated by (9). When the selected length for the CMF is greater than the actual length of the channel, it contributes to provide better ISI cancelation window for equalization, where the price is paid for more complexity. The error function in (19) provides a true identity for the equalization

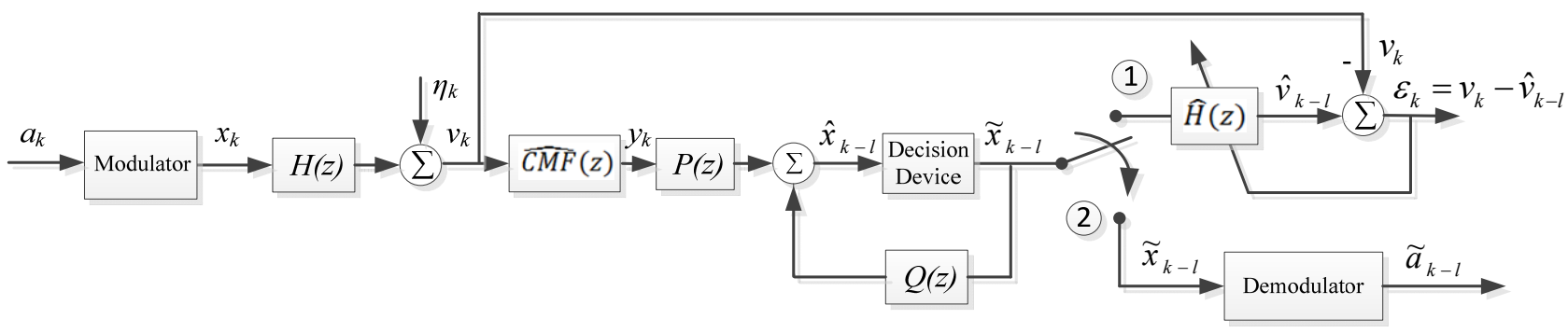

Fig. 2. The proposed system, switch is closed on "1" during the blind training and turned to position " 2 " for data recovery phase. 


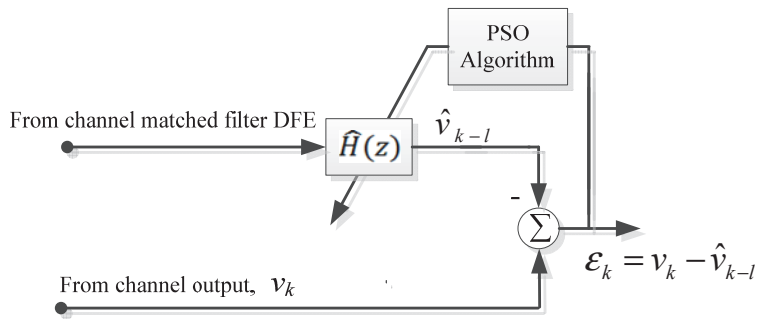

Fig. 3. The error circuit.

process of data $x_{k}$. Equation (19) can be configured as an MMSE type training process with a cost function

$$
J=\min \left\{\left|\varepsilon_{k}\right|^{2}\right\}
$$

The error calculation circuit is given in Fig. 3 where the coefficients of matched filter, $\widehat{C M F}(z)$ and estimated channel filter, $\hat{H}(z)$ are calculated by PSO algorithm. The proposed PSO algorithm is explained below.

First operation of PSO is to assign random values for each particle where particles represent the CMF coefficient sets. Therefore, multiple starting points for the CMF coefficients are maintained by using multiple particles of PSO. The values of particles have been controlled whether or not they are within acceptable limit which is pre-defined for CMF coefficients. Then, at the output of channel, the output of matched filter is calculated using (15) and the output of equalizer is calculated by (10) for every individual particle. The estimated data related to each individual particle is used to calculate the error function and every particle (every set of CMF coefficients) is updated by using (12) and (13). PSO approximates the channel matched filter coefficients from different random starting points to the optimum values. Therefore it minimizes the error function and MSE as well. The fitness function considered in every iteration represents an estimation of MSE over the input window. At the $n$th iteration, the estimation of MSE for the $i$ th particle is given by (21).

$$
J_{i}(n)=\frac{1}{N} \sum_{i=1}^{N}\left|\varepsilon_{j i}(n)\right|^{2}
$$

where $N$ is the length of the window of the input data and $\varepsilon_{i j}(n)$ is the $j$ th error for the $i$ th particle. When $\widehat{C M F}(z)$ and $H(z)$ coefficients are adaptively optimized by controlling the trend of the error value for the best performing particle, the training is terminated and the operation is switched to data recovery processes.

\section{Computer Simulation Results}

In this section, the performance analysis of proposed PSO-CMF-DFE is given. The simulation results are obtained over 2000 Monte-Carlo type packet averaging. Every packet containing 4000 QPSK modulated symbols are transmitted by 2000 different channels where the channel profile is 3 taps Proakis's B type channel [25]. The RMS delay spread of channel is $42 \mathrm{~ns}$ and bandwidth of the channel is $24.5 \mathrm{MHz}$. In order to cover all channel profile, the size of the estimated channel profile is selected as $L^{\prime}=5$ at the receiver. A DFE filter with $L_{\mathrm{ff}}=5$ taps of feedforward and $L_{\mathrm{fb}}=4$ taps of feedback filter are used for equalization. The performance of proposed PSO-CMFDFE has been compared with those using LMS-CMF-DFE and CMA-DFE algorithms. In the LMS-CMF-DFE, the blind training is carried out using the error function given by (19). In this study, the implemented PSO algorithm is the classic one and first presented by [23]. In our simulation 40 different particles are used for PSO implementation where the problem constraint space is $[-1.5,1.5]$ for initializing the particles. This limitation leads to constraint the channel coefficients within minimum of -1.5 and maximum of 1.5. The other parameters used in the algorithms are given in Tab. 1.

\begin{tabular}{|c|c|c|c|c|}
\hline CMA-DFE & LMS-CMF-DFE & \multicolumn{3}{|c|}{ PSO-CMF-DFE } \\
\hline$\mu$ & $\mu_{\text {CMF-DFE }}$ & $c_{1}$ & $c_{2}$ & $N$ \\
\hline 0.0075 & 0.02 & 1.5 & 2.0 & 150 \\
\hline
\end{tabular}

Tab. 1. Algorithm parameter settings in simulations.

The MSE performances of the algorithms are given by Fig. 4 and Fig. 5, and the BER performances are given in Fig. 6 and Fig. 7. Figure 4 and Figure 5 show the convergence rate of the algorithms in low SNR and high SNR values, respectively. For low SNR values given in Fig. 4, where SNR is equal to $15 \mathrm{~dB}$, the MSE performances of LMS-CMF-DFE and proposed PSO-CMF-DFE are much better than the performance of conventional CMA-DFE algorithm. The error floors of both LMS-CMF-DFE and PSO-CMF-DFE has decreased below $M S E=1.5 \times 10^{-2}$ and almost stabilized after 600 training iterations. However, as it is shown in Fig. 4, the proposed PSO based algorithm starts with high error level but quickly decreases below the other trainings within 50 to 100 training iterations and keeps performing better. The final error floor for the proposed algorithm is $M S E=7 \times 10^{-3}$ which is acceptable for many high performance applications when the SNR is lower than $15 \mathrm{~dB}$.

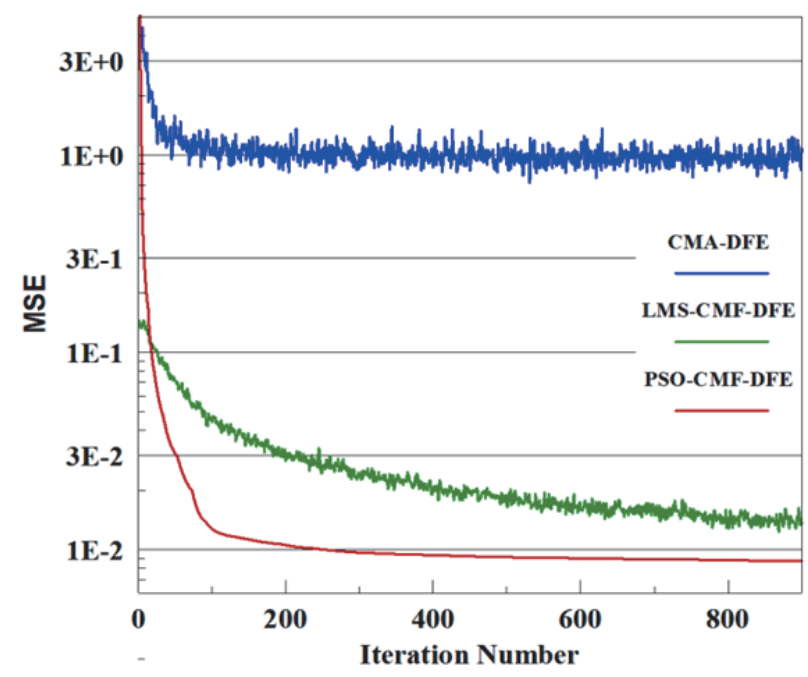

Fig. 4. MSE performance of the blind adaptive channel equalizers for an SNR of $15 \mathrm{~dB}$ in a stationary environment. 


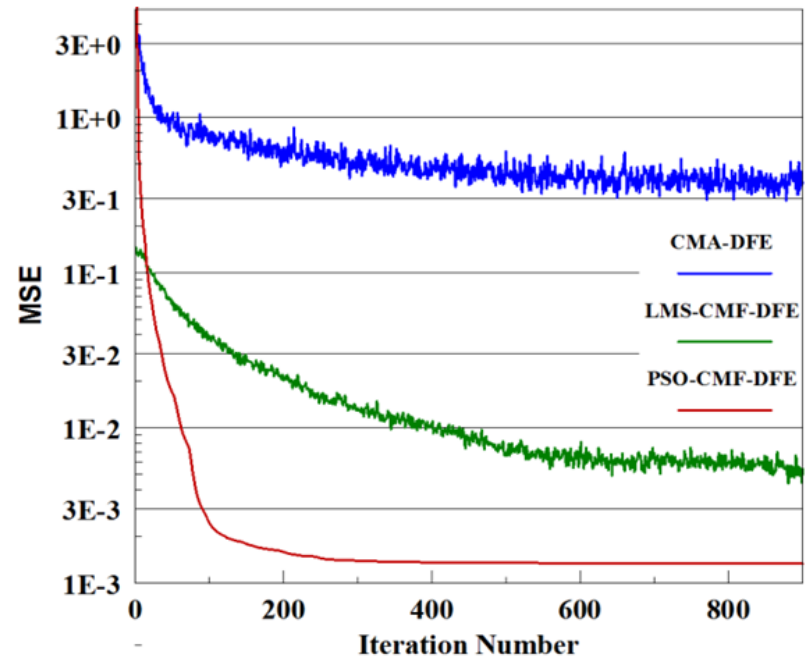

Fig. 5. MSE performance of the blind adaptive channel equalizers for an SNR of $30 \mathrm{~dB}$ in a stationary environment.

Figure 5 presents the MSE performances of simulated algorithms in higher SNR region, where $S N R=30 \mathrm{~dB}$. Similar performance comparisons can also be made in low SNR region presented in Fig. 4. But, here the error floor of proposed PSO-CMF-DFE gets into the stable region after 300 training iterations, having the minimum MSE of $1.3 \times 10^{-3}$ which is unexpectedly low. The performances of LMS-CMF-DFE and CMA-DFE get better when the SNR gets higher, but their performances are still much lower than the performance of the proposed method.

So far, the error floor of blind equalizations has been found too high to take place in practical high performance applications. Therefore, they are not able to compete with those using a training sequence based non-blind equalizations. However, the presented results show that the convergence rate of proposed PSO-CMF-DFE is quite higher. Furthermore, the error floor of the proposed method is comparable to the performances of training based algorithms as shown in Fig. 7. Therefore, the BER performance analysis of algorithms can be carried out for comparisons of blind and non-blind algorithms.

Whatever the training algorithm is, the phase information of a coherent modulation technique, such as QPSK, cannot be recovered [19]. So, a CAZAC sequence of 64 symbols is placed at the beginning of the data symbols in order to recover the phase information which is required to calculate BER performances. This CAZAC sequence is not used for trainings and can be considered as the available information in simulations only. Figure 6 shows the BER performances of blind equalization algorithms. The obtained BER performances are compatible with the MSE performances given in Fig. 4 and Fig. 5. It can be easily seen that the proposed method outperforms the performances of conventional CMA-DFE and LMS-CMF-DFE.

BER performances of blind (LMS-CMF-DFE, PSOCMF-DFE) and non-blind (LMS-DFE, PSO-DFE) equalization algorithms are given in Fig. 7. The BER performance graphics are obtained after 196 iterations for blind

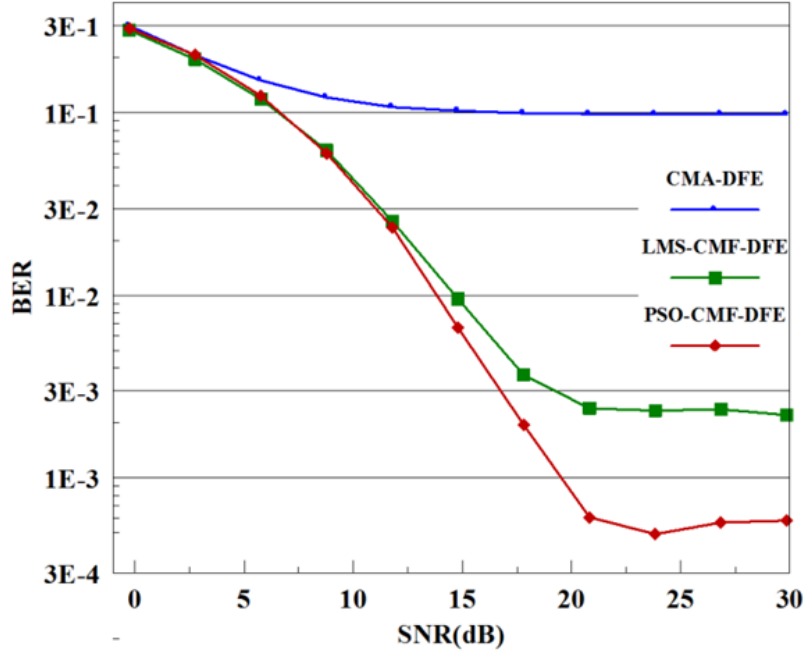

Fig. 6. BER performance results of the blind adaptive channel equalizers.

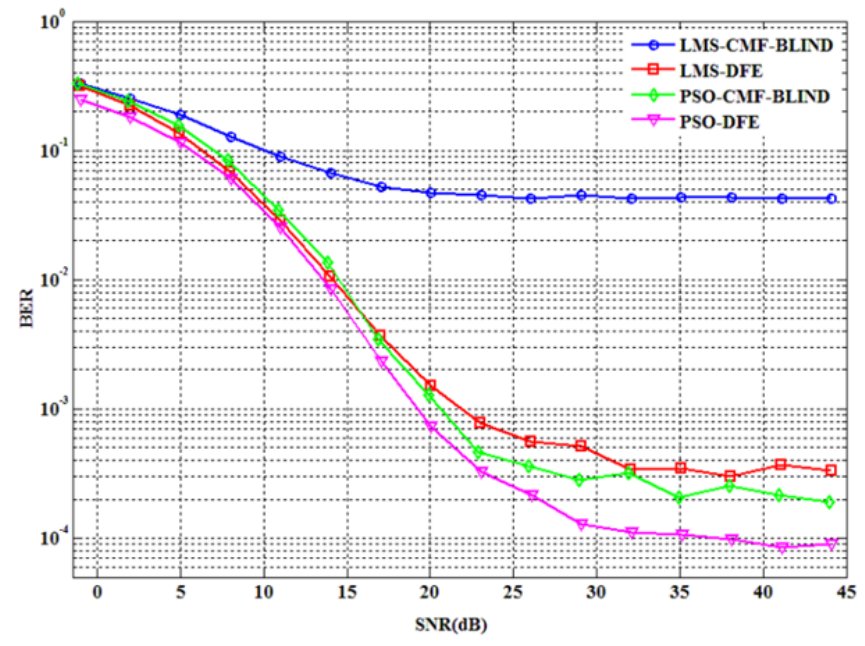

Fig. 7. BER performance results of the blind and non-blind adaptive channel equalizers.

and non-blind trainings. As it can be seen from the performance graphics, LMS-CMF-DFE has a poor performance because of the low convergence speed. As expected, the conventional LMS-DFE equalizer has higher performance than the LMS-CMF-DFE equalizer. It can be observed that while LMS-CMF-DFE equalizer convergences to $4.2 \times 10^{-2}$ error floor, LMS-DFE equalizer convergences to $3.0 \times 10^{-4}$ error floor. When the PSO algorithm is used as the adaptive algorithm, PSO-CMF-DFE equalizer has higher performance than LMS-DFE equalizer because of its high convergence speed and low steady state error floor. PSOCMF-DFE equalizer convergences to $2.0 \times 10^{-4}$ error floor. As expected, PSO-DFE has the highest performance and convergences to $1.0 \times 10^{-4}$ error floor in steady state.

\section{Conclusions}

In this study, a new blind equalization method is proposed by using pre-calculated DFE coefficients, error calculations at the output of channel and PSO algorithm for high performance trainings. Here, the DFE coefficients are 
obtained blindly at the beginning of training by using the autocorrelation coefficients. Therefore, the blind training is carried out only to estimate the channel or its matched filter coefficients, which requires implementing linear system parameter estimation. The PSO has provided high speed convergence to complete the whole blind equalization process and produced low error level in this high performance blind receiver architecture. Since the proposed method transforms the blind channel equalization problem to the linear filter estimation problem, its performance is much better than the conventional blind trainings, i.e. CMA. The proposed method may be used as an alternative to training sequence based non-blind equalization techniques.

\section{References}

[1] TURIN, G.L., CLAPP, F.D., JOHNSTON, T.L., FINE, S.B. LAVRY, D. A statistical model of urban multipath propagation. IEEE Transactions on Vehicular Technology, 1972, vol. 21, no. 1, p. 1-9. DOI: 10.1109/T-VT.1972.23492

[2] LABAT, J., MACCHI, O., LAOT, C. Adaptive decision feedback equalization: Can you skip the training period? IEEE Transactions on Communications, 1998, vol. 46, no. 7, p. 921-930. DOI: $10.1109 / 26.701319$

[3] WERNER, J. J., YANG, J., HARMAN, D. D., DUMONT, G. A. Blind equalization for broadband access. IEEE Communication Magazine, 1999, vol. 37, no. 4, p. 87-93, DOI: 10.1109/35.755455

[4] YANG, J., WERNER, J. J., DUMONT, G. A. The multimodulus blind equalization and its generalized algorithms. IEEE Journal on Selected Areas in Communication, 2002, vol. 20, no. 5, p. 997-1015. DOI: 10.1109/JSAC.2002.1007381

[5] SATO, Y. A method of self-recovering equalization for multilevel amplitude modulation systems. IEEE Transactions on Communications, 1975, vol. 23, no. 6, p. 679-682. DOI: 10.1109/TCOM.1975.1092854

[6] GODARD, D. Self-recovering equalization and carrier tracking in two dimensional data communications systems. IEEE Transactions on Communications, 1980, vol. 28, no. 11, p. 1867-1875. DOI: $10.1109 /$ TCOM.1980.1094608

[7] TREICHLER, J.R., AGEE, B. G. A new approach to multipath correction of constant modulus signals. IEEE Transactions on Acoustics, Speech, and Signal Processing, 1983, vol. 31, no. 2, p. 459-472. DOI: $10.1109 /$ TASSP.1983.1164062

[8] GOUPIL, A., POLICOT, J. An efficient blind decision feedback equalizer. IEEE Communication Letters, 2010, vol. 14, no. 5, p. 462-464. DOI: 10.1109/LCOMM.2010.05.092150

[9] CHEN, S. Low complexity concurrent constant modulus algorithm and soft decision directed scheme for blind equalization. IEE Proceedings-Visual, Image, Signal Processing, 2003, vol. 150, no. 5, p. 312-320. DOI: $10.1049 /$ ip-vis:20030619

[10] CHAHED, I., BELZILE, J., KOUKI, A. B. Blind decision feedback equalizer based on high order MCMA. In Canadian Conference on Electrical and Computer Engineering. Ontario (Canada), 2004, vol. 4, p. 2111-2114. DOI: 10.1109/CCECE.2004.1347658

[11] FILHO, J. M., MIRANDA, M. D., SILVA, M. T. M. An efficient algorithm for decision feedback blind equalization of QAM signals. Revista Telecomunicacoes, 2011, vol. 13, no. 2, p. 79-86. ISSN: $2237-8820$

[12] DE CASTRO, F.C.C., DE CASTRO, M.C.F., ARANTES, D.S. Concurrent blind deconvolution for channel equalization. In IEEE
International Conference on Communication. Helsinki (Finland), 2001, vol. 2, p. 366-371. DOI: 10.1109/ICC.2001.936964

[13] OH, K. N., CHIN, Y. O. modified constant modulus algorithm: Blind equalization and carrier phase recovery algorithm. In IEEE International Conference on Communication. Seattle (WA), 1995, vol. 1, p. 498-502. DOI: 10.1109/ICC.1995.525219

[14] JONES, D. L. A normalized constant modulus algorithm.In IEEE Conference Record of the Twenty-Ninth Asimolar Conference on Signals, Systems and Computers. Pacific Grove (USA), 1996, vol. 1, p. 694-697. DOI: 10.1109/ACSSC.1995.540639

[15] NASIR, A. A., DURRANI, S., KENNEDY R. A. Modified Constant Modulus Algorithm for joint blind equalization and synchronization. In Australian Communication Theory Workshop (AusCTW 2010). Canberra (ACT), 2010, p. 56-64. DOI: 10.1109/AUSCTW.2010.5426762

[16] HATZINAKOS, D., NIKIAS, C. L. Blind equalization using a tricepstrum-based algorithm. IEEE Transactions on Communications, 1991 , vol. 39 , no. 5 , p. 669-682. DOI: $10.1109 / 26.87158$

[17] TONG, L., XU, G., HASSIBI, B., KAILATH, T. Blind channel identification based on second-order statistics: A frequencydomain approach. IEEE Transactions on Information Theory, 1995, vol. 41, no. 1, p. 329-334. DOI: 10.1109/18.370088

[18] SESHADRI, N. Joint data and channel estimation using fast blind trellis search techniques. IEEE Transactions on Communications, 1994, vol. 42, no. 234, p. 1000-1011. DOI: 10.1109/TCOMM.1994.580208

[19] BAYKAL, B. Blind matched filter receiver. IEEE Transactions on Circuits and Systems-I: Regular Papers, 2004, vol. 51, no. 2, p. 379-390. DOI: 10.1109/TCSI.2003.820238

[20] BAYKAL, B. Blind channel estimation via combining autocorrelation and blind phase estimation. IEEE Transactions on Circuits and Systems-I: Regular Papers, 2004 vol. 51, no. 6, p. 1125-1131. DOI: 10.1109/TCSI.2004.829235

[21] COŞKUN, A., KALE, İ. All-adaptive blind matched filtering for the equalization and identification of multipath channels: A practical approach. IEEE Transactions on Circuits and Systems I: Regular Papers, 2013, vol. 60, no. 1, p. 232-242. DOI: 10.1109/TCSI.2012.2215711

[22] KAYA, I., TUGCU, E., ÖZEN, A., NIX, A. R. Fast convergence algorithm for blind channel estimation and equalization using CMF-DFE. In $81^{s t}$ Vehicular Technology Conference (VTC Spring). Glasgow (UK), 2015, p. 1-7, DOI: 10.1109/VTCSpring.2015.7145839

[23] KENNEDY, J., EBERHART, R. C. Particle swarm optimization. In Proceedings of the IEEE International Conference on Neural Networks. Perth, (Australia), 1995, vol. 4, p. 1942-1948. DOI: 10.1109/ICNN.1995.488968

[24] BALTACI, Y., KAYA, I., NIX, A. R. Implementation of a HIPERLAN/1 Compatible CMF-DFE equalizer. In Proceedings of the IEEE $51^{\text {st }}$ Vehicular Technology Conference (VTC 2000 Spring). Tokyo (Japan), 2000, vol. 3, p. 1884-1888. DOI: 10.1109/VETECS.2000.851599

[25] PROAKIS, J. G. Digital Communications. Fourth ed. Singapore: McGraw-Hill, 2001. ISBN: 978-0071181839

[26] HAYES, M. H. Statistical Digital Signal Processing and Modeling. New York: John Wiley \& Sons, Inc., 1996. ISBN: 9780471594314

[27] CIOFFI, J.M., DUDEVOIR, G.P., VEDAT EYUBOGLU, M., FORNEY, G. D. MMSE decision-feedback equalizers and coding: Part I: Equalization results. IEEE Transactions on Communications, 1995, vol. 43, no. 10, p. 2582-2594. DOI: 10.1109/26.469441

[28] KAYA, I. A study of high performance equalization and preequalization for high speed wireless data communications. Ph.D. Thesis, University of Bristol, April 1998. 
[29] LING, S. H., LU, H. H. C., CHAN, K. Y., LAM, H. K. Hybrid particle swarm optimization with wavelet mutation and its industrial applications. IEEE Transactions on Systems, Man, and Cybernetics, Part B: Cybernetics, 2008, vol. 38, no. 3, p. 743-763. DOI: 10.1109/TSMCB.2008.921005

[30] COELHO, L. D., GUERRA F. A. Applying particle swarm optimization to adaptive controller. In Soft Computing in Industrial Applications, 2007, vol. 39, p. 82-91. DOI: 10.1007/978-3-54070706-6_8

[31] AL-AWAMI, A. T., ZERGUINE, A., CHEDED, L., ZIDOURI, A., SAIF, W. A new modified particle swarm optimization for adaptive equalization. Digital Signal Processing, 2011, vol. 21, no. 2, p. 195-207. DOI: 10.1016/j.dsp.2010.05.001

[32] ZUBAIR, M., CHOUDHRY, M. A. S., NAVEED, A., QURESHI, I. M. Joint channel and data estimation using particle swarm optimization. IEICE Transactions on Communications, 2008, vol. E91.B, no. 9, p. 3033-3036. DOI: 10.1093/ietcom/e91b. 9.3033

[33] TORRES, G. V. CMA equalization with decision-directed phase correction. In Conference Record of the Thirty-Eight Asilomar Conference on Signals, Systems and Computers. Monterey (USA), 2004, vol. 2, p. 2047-2050. DOI: 10.1109/ACSSC.2004.1399525

\section{About the Authors ...}

Emin TUGCU received B.S., M.Sc. and Ph.D. degrees in the Dept. of Electrical and Electronics Engineering from
KTU in 2004, 2007 and 2014 respectively. He has been working as a lecturer (Assist. Prof.) in the Dept. of Electronics and Communication Engineering since 2015. His current research interests are signal processing, wireless communication, channel equalization, and coding theory. $\mathrm{He}$ is a member of IEEE.

Ismail KAYA has graduated from KTU, and completed Ph.D. in 1998 in the University of Bristol (UoB). He has been working as a lecturer (Assoc. Prof.) in the Dept. of Electrical and Electronics Engineering since 1999. His academic interests are on signal processing, channel contour measures, equalization, blind equalization, OFDM receivers, and MIMO. He has established a research lab. at KTU and completed three patent applications on the area of fast equalization techniques in UoB.

Ayhan YAZGAN received the B.S. and M.Sc. degree in Electrical and Electronics Engineering from the KTU in 2005 and 2008 respectively. He received his second M.Sc. degree in the program of Microelectronics and Photonics from Halmstad University, Sweden in 2011. He received the Ph.D. degree in 2015 from KTU. He has been working as an Assit. Prof. in the Dept. of Electrical and Electronics Engineering since 2015. His current research interests are reconfigurable antennas, optical OFDM systems, optical channel estimation, and RoF systems. 